\title{
ESTUDIO EXPERIMENTAL DE LA DEGRADACIÓN DEL PLAGUICIDA METOMILO EN AGUAS SUPERFICIALES APLICANDO PROCESOS AVANZADOS DE OXIDACIÓN
}

\author{
Grecia Quispe Suni ${ }^{\mathrm{a}}$, Cynthia Medina Flores ${ }^{\mathrm{a}}$, Andrea Villasante Aparicio ${ }^{\mathrm{a}}$, \\ Julian Rengifo Herrera ${ }^{\mathrm{b}}$, Miriam Bernal de Arenas ${ }^{\mathrm{c}^{*}}$
}

\begin{abstract}
RESUMEN
El uso intensivo de agroquímicos para la producción de alimentos en los países en desarrollo ha generado un problema de interés ambiental pues muchas de estas sustancias que suelen ser difíciles de biodegradar pueden alcanzar fuentes naturales generando su contaminación. Es por ello que la búsqueda de nuevas tecnologías para la eliminación de estos contaminantes ha surgido con mucho interés. Los procesos avanzados de oxidación (PAOs), cuya principal característica es la generación de especies altamente oxidantes como el radical hidroxilo, pueden lograr la modificación química del contaminante generando subproductos que pueden ser menos dañinos con el medio ambiente. En este trabajo se evaluó la degradación de metomilo, un insecticida altamente utilizado en la región de Arequipa, Perú, utilizando diferentes PAOs como la ozonización en medio alcalino, UV-C/H2O2 y procesos foto-Fenton a pH ácido. Se evaluó a escala de laboratorio el efecto de matriz de aniones como bicarbonatos, fluoruros y nitratos y posteriormente en aguas naturales superficiales. Los resultados indican que la mayor eliminación de metomilo en agua real se logró en $99.38 \%$ bajo UV-C $/ \mathrm{H}_{2} \mathrm{O}_{2}$ seguida del proceso fotofenton, con un $94.12 \%$ de degradación y la ozonólisis a pH alcalino ( $\mathrm{pH} 11$ ) con un $40 \%$ de degradación, demostrando que los PAOs evaluados pueden ser una alternativa promisoria para eliminar agroquímicos de fuentes naturales.
\end{abstract}

Palabras clave: Ozonólisis, foto-Fenton, $\mathrm{UV}-\mathrm{C} / \mathrm{H}_{2} \mathrm{O}_{2}$, metomilo, PAOs

\section{EXPERIMENTAL STUDY OF THE DEGRADATION OF THE PESTICIDE METOMIL IN SURFACE WATERS APPLYING ADVANCED OXIDATION PROCESSES}

\begin{abstract}
The intensive use of agrochemicals for food production in developing countries has generated a problem of environmental concern since many of these substances, which are usually difficult to biodegrade, can reach natural sources, generating their contamination. That is why the search for new technologies for the elimination of these pollutants has arisen with great

\footnotetext{
a Facultad de Ingeniería de Procesos, Departamento de Ingeniería Metalúrgica y Ambiental, Universidad Nacional de San Agustín de Arequipa, Av. Independencia S/N., Perú.

b Facultad de Ciencias Exactas, Departamento de Química, UNLP-CCT La Plata, Buenos Aires, Argentina

c Facultad de Ciencias Naturales y Formales, Departamento Académico de Química. Universidad Nacional de San Agustín de Arequipa, Av. Independencia S/N. Perú, *mbernalt@unsa.edu.pe
} 
interest. Advanced oxidation processes (AOP), whose main characteristic is the generation of highly oxidizing species such as the hydroxyl radical, can achieve the chemical modification of the pollutant, generating by-products that can be less harmful to the environment. In this work, the degradation of methomyl, a highly used insecticide in the Arequipa region, Peru, was evaluated using different PAOs such as ozonation in alkaline medium, UV-C/ $\mathrm{H}_{2} \mathrm{O}_{2}$ and photo-Fenton processes at acid $\mathrm{pH}$ in natural surface waters. The effect of anion matrix such as bicarbonates, fluorides and nitrates were evaluated on a laboratory scale and later in natural surface waters. The results indicate that the highest methomyl removal in real water was achieved at $99.38 \%$ for $\mathrm{UV}-\mathrm{C} / \mathrm{H}_{2} \mathrm{O}_{2}$ followed by the photofenton process, with $94.12 \%$ degradation and ozonolysis at alkaline ( $\mathrm{pH} 11$ ) with $40 \%$ degradation, demonstrating that the evaluated PAOs can be a promising alternative to eliminate agrochemicals from natural sources.

Key words: Ozonolysis, photo-Fenton, $\mathrm{UV}-\mathrm{C} / \mathrm{H}_{2} \mathrm{O}_{2}$, methomyl, AOP

\section{INTRODUCCIÓN}

El metomilo $\left(\mathrm{C}_{5} \mathrm{H}_{10} \mathrm{~N}_{2} \mathrm{O}_{2} \mathrm{~S}\right)$ es un insecticida, perteneciente a la familia de los carbamatos y es muy utilizado en el Perú para el control de diversas plagas agrícolas ${ }^{1}$. Este insecticida posee características fisicoquímicas relacionadas a su solubilidad en agua $(58 \mathrm{mg} / \mathrm{L})$ y la toxicidad que puede causar fácilmente contaminación del agua en áreas agrícolas. Por otro lado, los procesos convencionales de biotratamiento presentan limitaciones para la remoción de metomilo en agua, aunque algunos trabajos han reportado el uso de cepas bacterianas específicas como Paracoccus, Pseudomonas, Aminobacter, Flavobacterium, Alcaligenes, Bacillus, Serratia, Novosphingobium, y Trametes ${ }^{2}$.

Diferentes estudios sobre procesos para la remoción de metomilo se han concentrado en el uso de procesos avanzados de oxidación fotoquímicos y no fotoquímicos como el proceso Fenton, Foto-Fenton ${ }^{3,4}$ y fotocatálisis con $\mathrm{TiO}_{2}^{5,6}$. El proceso fotofenton es un método que emplea un catalizador y sales de hierro que, bajo irradiación con luz ultravioleta o solar, facilitan su recuperación y reutilización, a la vez que reaccionan con peróxido de hidrógeno para la liberar $\mathrm{OH}$, que oxidan a compuestos orgánicos.El proceso $\mathrm{H}_{2} \mathrm{O}_{2} / \mathrm{UV}$ se basa en la producción de un oxidante efectivo, el radical hidroxilo $(\bullet \mathrm{OH})$, a través de la irradiación del peróxido de hidrógeno con radiación ultravioleta, según la reacción $1^{7}$.

$$
\mathrm{H}_{2} \mathrm{O}_{2}+\mathrm{hv} \rightarrow 2 \cdot \mathrm{OH} \quad \Delta \mathrm{G}_{\mathrm{R}}=-237 \mathrm{~kJ} / \mathrm{mol}
$$

La descomposición fotoinducida del peróxido de hidrógeno en agua pura se racionaliza mediante el mecanismo de cadena radical Haber-Weiss, según el cual los radicales hidroxilos originados por el clivaje homolítico del enlace O-O inician una secuencia de reacciones caracterizadas por un paso de iniciación (reacción 1), seguido de un paso de propagación dado por las otras reacciones ${ }^{7}$. El proceso de Ozonización se basa en procesos fisicoquímicos que involucran la generación y uso de especies transitorias poderosas, principalmente el radical hidroxilo ${ }^{8}$. La ozonización ha sido exitosamente utilizada para la remoción de metomilo en agua Milli-Q. 
El objetivo de la presente investigación es estudiar la degradación de metomilo en aguas reales provenientes de drenes agrícolas del Sector Uraca-Corire de la Provincia de Arequipa, utilizando tres Procesos Avanzados de oxidación: Ozonización, Foto Fenton y Peróxido de hidrógeno y luz ultravioleta; de modo que se verifique la eficiencia de cada uno de estos procesos para recomendar el proceso óptimo para el tratamiento del mencionado plaguicida.

\section{PARTE EXPERIMENTAL}

\subsection{Reactivos del proceso foto Fenton y H2O2/UV y Ozonización}

En todos los experimentos se utilizaron los siguientes reactivos: peróxido de hidrógeno $\left(\mathrm{H}_{2} \mathrm{O}_{2}\right)$ PANREAC ${ }^{\circledR}, 30 \%(\mathrm{~V} / \mathrm{V})$, Metomilo $(\mathrm{C} 5 \mathrm{H} 10 \mathrm{~N} 2 \mathrm{O} 2 \mathrm{~S})$; con una pureza $\geq 98 \%$, SIGMAALDRICH ${ }^{\circledR}$, ácido sulfúrico, $\mathrm{H}_{2} \mathrm{SO}_{4} 6 \mathrm{~N}$, hidróxido de sodio $(\mathrm{NaOH})$, ácido clorhídrico $\mathrm{HCl}$ , sulfato ferroso heptahidratado, $\left(\mathrm{FeSO}_{4} \cdot 7 \mathrm{H}_{2} \mathrm{O}\right)$ HIMEDIA ${ }^{\circledR}, 99.5 \%$, nitrato de sodio $\mathrm{AR} /$ ACS $\left(\mathrm{NaNO}_{3}\right.$ ), de la marca Central Drug House (P) Ltd. con una pureza del 99,5-99,8 \%, carbonato de calcio $\left(\mathrm{CaCO}_{3}\right)$, de la marca Central Drug House (P) Ltd. con una pureza del $99,5 \%$, cloruro de sodio de marca Central Drug House (P) Ltd, Yoduro de potasio al 2\%. KI Merck ${ }^{\circledR}$

\subsection{Sistemas de proceso Foto Fenton, $\mathrm{H}_{2} \mathrm{O}_{2} / \mathrm{UV}$ y Ozonización}

Para los tratamientos realizados con el proceso foto-Fenton y peróxido con UV, se dispuso de radiación artificial con lámparas tubulares fluorescentes de vapor de mercurio de baja presión Actinic BL TL TL-D marca Philips, 18W/10 1SL/25, las cuales se ubicaron en el interior de una cámara de acero inoxidable iluminando desde la parte superior, así mismo se contó con un sistema de sensores de temperatura y un protector anti-UV, traslucida en la cara anterior, un agitador magnético y un vaso reactor de cuarzo, que permite el paso de la luz hacia la solución (Figura 1).

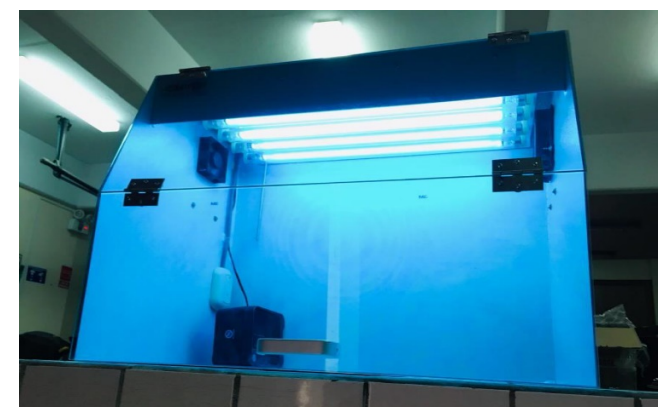

Figura 1. Cámara real de reacción. Fuente Propia. Muestra del diseño real estructurado para las experimentaciones acoplado de un sistema de lámparas UV-A, Actinic BL TL-D 18W/10 1SL/25, marca PHILIPS. 
El ozono se genera a partir de oxígeno comercial mediante un generador de ozono (Guolin, modelo CF-G-3-20g). El flujo del oxidante se dirige hacia un reactor de vidrio pyrex de $500 \mathrm{~mL}$ que contiene la muestra a tratar donde se burbujeo continuamente el reactor por medio de un difusor de vidrio poroso. Posteriormente se dirige hacia un reactor posterior (trampa de ozono) que contiene una solución de yoduro de potasio al $2 \%$. La concentración de ozono se ajustó mediante el método Indigo ${ }^{9}$. Para la experimentación se utilizó metomilo de marca Sigma-Aldrich. La experimentación fue llevada en tres etapas de acuerdo con el tipo de agua utilizada: agua destilada, agua simulada y agua real.

\subsection{Determinación de concentraciones óptimas para la degradación de metomilo}

Para los experimentos foto Fenton se desarrolló un diseño experimental de factores independientes para determinar la dosis óptima de las variables (tiempo, concentración de $\mathrm{Fe}+2 / \mathrm{H} 2 \mathrm{O} 2$ y $\mathrm{pH}$ ) a intervenir en la degradación del metomilo, para lo cual en cada experimentación se realizó el diseño experimental basado en la ecuación 2 , donde " $t$ " es la cantidad de tratamientos, "r" el número de repeticiones y "N" el número de análisis:

$$
t x r=N
$$

Para los experimentos con $\mathrm{H}_{2} \mathrm{O}_{2} / \mathrm{UV}$, el diseño experimental que se desarrolló fue de factores independientes para la degradación de metomilo en agua destilada (condiciones óptimas), agua simulada y agua real muestra con el proceso peróxido de hidrógeno y luz ultravioleta $\left(\mathrm{H}_{2} \mathrm{O}_{2} / \mathrm{UV}\right)$. En todos los experimentos se trabajó con una concentración inicial de $10 \mathrm{mg} / \mathrm{L}$ de metomilo, se comenzó con la determinación del tiempo óptimo teniendo en cuenta cinco tiempos en el último experimento $(0,1,2,4$ y $8 \mathrm{~min})$, seguido de la determinación de la concentración de peróxido de hidrógeno óptima considerando dos concentraciones $3 \mathrm{mg} / \mathrm{L}$ y $10 \mathrm{mg} / \mathrm{L}$, para terminar, se hizo la determinación del tipo de radiación ultravioleta UV-C, UV-B y UV-A. Se realizaron tres repeticiones en todos los experimentos.

Para los experimentos con ozonización, se determinó las condiciones óptimas a través de un diseño experimental, (ver Tabla 1) para las variables $\mathrm{pH}$ y tiempo de contacto. Se estableció como un parámetro fijo el $\mathrm{pH}$ natural de las muestras de agua de los drenes objeto de estudio. Teniendo en cuenta que valores altos de $\mathrm{pH}$ aumenta la eficiencia en la degradación de los contaminantes orgánicos ${ }^{8}$ se seleccionó un valor de $\mathrm{pH}$ de 11. En el caso donde se ajustó el pH, este se realizó con $\mathrm{NaOH} 0.1 \mathrm{M}$. Los niveles establecidos para el tiempo de contacto fueron seleccionados tomando en cuenta los resultados obtenidos en los ensayos preliminares. Los experimentos realizados a $\mathrm{pH} 8$ utilizaron tiempos de contacto de 5, 10, 15, 30 min mientras que a pH 11 se establecieron intervalos de tiempo de 2, 4, 8, $10 \mathrm{~min}$. La concentración de ozono que se utilizó está limitada por el equipo y corresponde a la mínima concentración alcanzada de $0.07 \mathrm{mg} / \mathrm{L}$ a un flujo constante de $0.5 \mathrm{~L} / \mathrm{min}$. La concentración inicial de Metomilo de estos experimentos fue de $10 \mathrm{mg} / \mathrm{L}$. 
Tabla 1. Diseño experimental para pH 8 y pH 11.

\begin{tabular}{ccll}
\hline Tratamientos & $\begin{array}{l}\text { Concentración } \\
\text { de Ozono }(\mathbf{m g} / \mathbf{L})\end{array}$ & $\mathbf{p H}$ (unidades) & $\begin{array}{l}\text { Tiempo } \\
(\mathbf{m i n})\end{array}$ \\
\hline $\mathbf{1}$ & 0.07 & Natural (8.0) & 5 \\
$\mathbf{2}$ & 0.07 & Natural (8.0) & 10 \\
$\mathbf{3}$ & 0.07 & Natural (8.0) & 15 \\
$\mathbf{4}$ & 0.07 & Natural (8.0) & 30 \\
$\mathbf{5}$ & 0.07 & Modificado (11.0) & 2 \\
$\mathbf{6}$ & 0.07 & Modificado (11.0) & 4 \\
$\mathbf{7}$ & 0.07 & Modificado (11.0) & 8 \\
$\mathbf{8}$ & 0.07 & Modificado (11.0) & 10 \\
\hline
\end{tabular}

\subsection{Caracterización fisicoquímica de las aguas reales de los drenes del sector de Uraca- Corire}

Las aguas residuales caracterizadas provienen de los drenes agrícolas del valle de Majes, distrito de Uraca-Corire, provincia y departamento de Arequipa, Perú. Se establecieron 5 estaciones de muestreo (Tabla 2) para la caracterización de fisicoquímica de aniones; cloruros, fluoruros, sulfatos, fosfatos y carbonatos del agua real.

Tabla 2. Estaciones de muestreo de aguas superficiales en Uraca-Corire.

\begin{tabular}{ccccc}
\hline \multirow{2}{*}{ Código } & Nombre de muestra & \multicolumn{3}{c}{ Coordenadas UTM WGS 84 } \\
\cline { 3 - 5 } & & Zona & Este & Norte \\
\hline P-01 & Efluente de la Zona 1 & $18 \mathrm{~K}$ & $769974 \mathrm{~m} \mathrm{E}$ & $8206519 \mathrm{~m} \mathrm{~S}$ \\
P-02 & Efluente de la Zona 2 & $18 \mathrm{~K}$ & $770047 \mathrm{~m} \mathrm{E}$ & $8206452 \mathrm{~m} \mathrm{~S}$ \\
P-03 & Confluente de la zona 3 & $18 \mathrm{~K}$ & $769701 \mathrm{~m} \mathrm{E}$ & $8205290 \mathrm{~m} \mathrm{~S}$ \\
P-04 & Efluente de la Zona 4 & $18 \mathrm{~K}$ & $769977 \mathrm{~m} \mathrm{E}$ & $8205073 \mathrm{~m} \mathrm{~S}$ \\
P-05 & Confluente de la zona 5 & $18 \mathrm{~K}$ & $770585 \mathrm{~m} \mathrm{E}$ & $8200323 \mathrm{~m} \mathrm{~S}$ \\
\hline
\end{tabular}

La estación de muestreo P-05, colector final de los drenes, fue el punto para recolectar el agua para la experiencia con agua real. 


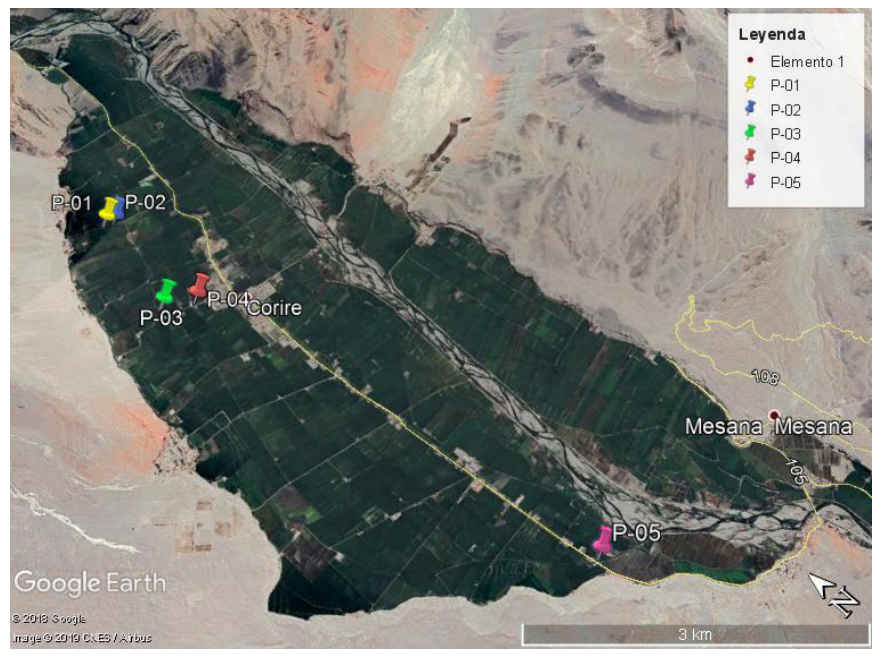

Figura 2. Distribución geográfica de los puntos de monitoreo. Adaptado de Google Earth.

\subsection{Metodología experimental para los ensayos Foto-Fenton, $\mathrm{H}_{2} \mathrm{O}_{2} / \mathrm{UV}$, y Ozonización} Para los ensayos Foto-Fenton se prepararon soluciones acuosas conteniendo $2.5 \mathrm{mg} / \mathrm{L}$ de Sulfato ferroso heptahidratado $\left(\mathrm{FeSO}_{4} \cdot 7 \mathrm{H}_{2} \mathrm{O}\right)$ disponible de HIMEDIA, el cual fue utilizado como fuente de $\mathrm{Fe}^{2+}$ y una concentración de metomilo de $10 \mathrm{mg} / \mathrm{L} \mathrm{El} \mathrm{pH}$ inicial de la solución fue ajustado a un valor de 3,6 utilizando $\mathrm{HCl}$ 0,1 M. Esta solución se colocó en un reactor de cuarzo con un volumen aproximado de $200 \mathrm{~mL}$. Esta solución fue colocada en la cámara de irradiación agregando previamente una concentración de $10 \mathrm{mg} / \mathrm{L}$ de $\mathrm{H}_{2} \mathrm{O}_{2}(30 \% \mathrm{v} / \mathrm{v}$ Panreac). La temperatura promedio durante los ensayos fue de $25^{\circ} \mathrm{C}$; la solución se agitó magnéticamente a 700-750 RPM. Los ensayos con agua superficial simulado contenían concentraciones de iones Fluoruro [F-] de $0.30 \mathrm{mg} / \mathrm{L}$, Nitrato [NO3- ] de $5 \mathrm{mg} / \mathrm{L}$ y Carbonato $\left[\mathrm{CO}^{2-}\right]$ de $20 \mathrm{mg} / \mathrm{L}$.

Los experimentos de $\mathrm{H}_{2} \mathrm{O}_{2} / \mathrm{UV}$ se llevaron a cabo en un reactor Batch utilizando un recipiente de cuarzo de $235 \mathrm{~mL}$. Las soluciones acuosas de metomilo (Sigma Aldrich) con una concentración de $10 \mathrm{mg} / \mathrm{L}$ a pH 5 se agitaron constantemente a 750 RPM por medio de un agitador magnético de cerámica (tipo SH-4C de AKMLAB) y fueron irradiadas en diferentes tiempos y diversas condiciones de concentración de peróxido de hidrógeno y tipo de radiación ultravioleta mediante el uso lámparas tubulares fluorescentes de radiación tipo UV-A, UV-B y UV-C y solución de $\mathrm{H}_{2} \mathrm{O}_{2}$ al $30 \% \mathrm{v} / \mathrm{v}$ (PANREAC) llevada a una concentración de 10 $\mathrm{mg} / \mathrm{L}$ para la realización de los ensayos. Todos los experimentos se realizaron a temperatura ambiente $\left(25^{\circ} \mathrm{C}\right)$.

Los tratamientos fueron llevados en tres etapas de acuerdo con tipo de agua utilizada: agua destilada, agua simulada y agua real. En la etapa de trabajo con agua destilada se realizó la determinación del tiempo, concentración de peróxido de hidrógeno y tipo de irradiación 
óptimos para posteriormente utilizar estos parámetros en los experimentos con agua simulada y agua real. En los tratamientos con agua simulada se trabajó con tres tipos diferentes de iones (carbonatos, nitratos y cloruros), con concentraciones de $20 \mathrm{mg} / \mathrm{L}, 5 \mathrm{mg} / \mathrm{L}$ y $60 \mathrm{mg} / \mathrm{L}$ respectivamente; con el fin de evaluar la existencia de alguna interferencia en la degradación de metomilo por parte de los aniones.

Las muestras de agua real recolectadas de los drenes agrícolas del distrito Uraca-Corire, fueron caracterizadas fisicoquímicamente tomando en cuenta los parámetros de temperatura, $\mathrm{pH}$, oxígeno disuelto, conductividad eléctrica, carbonatos, cloruros, fluoruros, nitratos, sulfatos y fosfatos. Las muestras se enriquecieron con $10 \mathrm{ppm}$ de metomilo y se sometieron al tratamiento de peróxido de hidrógeno y luz UV. Se realizaron dos experimentos control con el fin de determinar el efecto aislado del peróxido de hidrógeno y la radiación ultravioleta; y el efecto independiente de cada uno de los tipos de radiación (UV-A, UV-B, UV-C).

Para los ensayos de ozonización, se utilizó las condiciones óptimas obtenidas para realizar la experimentación en agua simulada. Se utilizó como matriz una solución de Metomilo a 10ppm (mg/L), en presencia de aniones: 60 ppm de Cloruros, 5 ppm de Nitratos, 20 ppm de Carbonatos, en agua simulada. Finalmente se utilizó como matriz, una solución de Metomilo a $10 \mathrm{ppm}(\mathrm{mg} / \mathrm{L})$ en agua real proveniente de los drenes de agricultura del Sector de UracaCorire. Evaluando la degradación de metomilo por cromatografía líquida acoplada a un detector de masas (LC-MS/MS).

\subsection{Análisis cromatográfico del metomilo}

Los análisis de residuos de plaguicidas se realizaron a través de Cromatografía Líquida y Cromatografía Gaseosa cada uno acoplado a un detector de Masas Triple Cuádruplo (GC MS/MS y LC - MS/MS). En diferentes intervalos de tiempo se extrajeron muestras para la cuantificación del metomilo residual, posteriormente se envió las muestras al laboratorio AGQ PERÚ SAC, con acreditación estándar ISO/IEC 17025:2005. El metomilo se analiza por la técnica de cromatografía líquida. La determinación de residuos de plaguicidas se basa en una extracción haciendo uso de la metodología QuEChERS ${ }^{10}$. Este método combina dos etapas, una de extracción de la muestra con acetonitrilo y diferentes sales. La columna utilizada es Columna C18 de $100 \mathrm{~mm}$ x 2'1 mm x 1'8 $\mu \mathrm{m}$, las fases móviles están compuestas por Fase A: Agua ( $0.05 \%$ ácido fórmico +2 molar formiato amónico) Fase B: Metanol $(0.05 \%$ acido fórmico); el volumen de inyección fue de $3 \mathrm{uL}$ para todas las muestras analizada con un flujo de $400 \mathrm{uL} / \mathrm{min}$. El detector usado es el EvoQ MS- Triple Cuadrupolo.

\section{RESULTADOS Y DISCUSIÓN}

\subsection{Caracterización del agua real}

La caracterización fisicoquímica del agua real (Tabla 3), muestra que el agua del Valle de Majes se caracteriza por tener un $\mathrm{pH}$ entre 7.88 a 8.99 (agua alcalina), con una conductividad entre 109 a $700 \mathrm{uS} / \mathrm{cm}$, una concentración de oxígeno disuelto entre 7.93-8.38 y una temperatura entre 18.6 - 20. Las mediciones para $\mathrm{pH}$ muestran que los puntos de muestreo 
P-01, P-02 y P-04, no cumplen con el Estándar Nacional de calidad del agua para la categoría 3 , debido a la presencia de especies carbonatadas, que elevan el $\mathrm{pH}$.

Tabla 3. Caracterización fisicoquímica de aguas provenientes de Uraca-Corire.

\begin{tabular}{|c|c|c|c|c|c|c|c|c|c|c|}
\hline \multirow[b]{2}{*}{ Zona } & \multicolumn{4}{|c|}{ Parámetros de campo } & \multicolumn{6}{|c|}{ Aniones mg/L } \\
\hline & $\begin{array}{l}\text { OD } \\
(\mathrm{mg} / \mathrm{L})\end{array}$ & $\begin{array}{l}\text { CE } \\
(\mu \mathrm{s} / \mathrm{cm})\end{array}$ & pH & $\begin{array}{l}\text { Temp } \\
\left({ }^{\circ} \mathrm{C}\right)\end{array}$ & $\mathrm{CO}_{3}{ }^{2-}$ & $\mathrm{Cl}^{-}$ & $\mathbf{F}^{-}$ & $\mathrm{NO}_{3}{ }^{-}$ & $\mathrm{SO}_{4}{ }^{2-}$ & $\mathrm{PO}_{4}{ }^{3-}$ \\
\hline P-01 & 8.36 & 700 & 8.93 & 19.7 & 37 & 57.0 & 0.59 & 3.2 & 156.9 & 0.80 \\
\hline P-02 & 8.34 & 698.5 & 8.99 & 20.0 & 33.5 & 55.8 & 0.34 & 2.6 & 143.8 & 0.58 \\
\hline P-03 & 7.93 & 878 & 8.12 & 19.5 & 9 & 63.5 & 0.25 & 5.3 & 0.56 & 197.3 \\
\hline P-04 & 8.06 & 830 & 8.51 & 19.9 & 18.5 & 63.8 & 0.27 & 4.2 & 185.5 & 0.79 \\
\hline P-05 & 8.23 & 1097 & 7.90 & 18.6 & 10 & 81.0 & 0.26 & 8.4 & 259.8 & 0.67 \\
\hline
\end{tabular}

En el análisis de aniones interferentes se muestra una concentración de Carbonatos (CO32) entre 9-33.7 mg/L, Cloruros (Cl-) entre 5.8-81 mg/L, Fluoruros (F-) entre 0.25-0.59 mg/L, Nitratos (NO3-) entre 2.6-8.4 mg/L, Sulfatos (SO42-) entre 0.56-259.8 mg/L y Fosfatos (PO43-) entre $0.58-197.3 \mathrm{mg} / \mathrm{L}$, todos los valores presentan diferencias entre punto y punto de muestreo.

\subsection{Degradación de metomilo en agua destilada proceso foto-fenton}

Para determinar el tiempo de reacción óptimo, la cinética de reacción se inicia con $\left[\mathrm{Fe}^{2+} /\right.$ $\left.\mathrm{H}_{2} \mathrm{O}_{2}\right]=2.5 / 10 \mathrm{mg} / \mathrm{L}, \mathrm{pH}=3.6$, e irradiación UV-A. Se evaluó el progreso de la degradación en tiempos de $0,5,15,30$ y 60 minutos para determinar la relación entre la concentración final de metomilo frente a la concentración inicial (Figura 3).

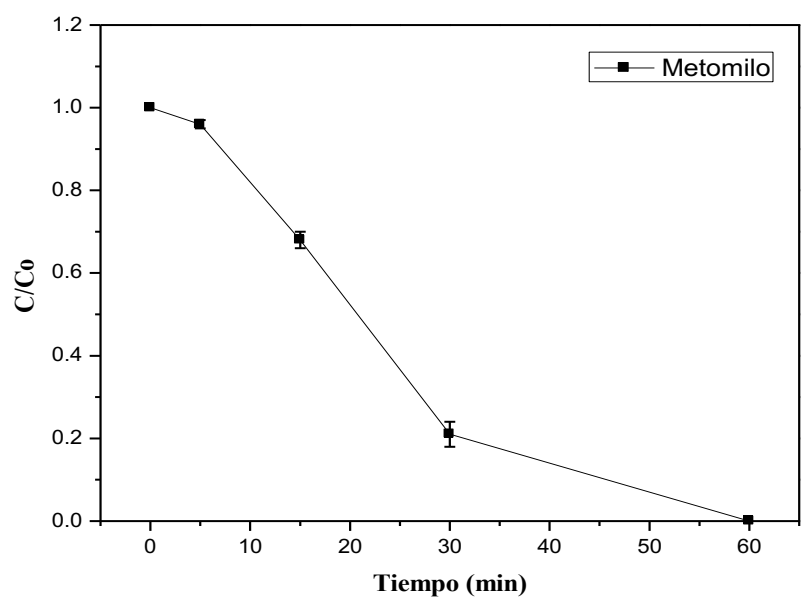

Figura 3. Relación de la concentración final frente a la concentración inicial de metomilo degradado por fotodegradación en el tiempo, bajo condiciones experimentales $\left[\mathrm{pH}=3.6, \mathrm{Fe}^{2+}=\right.$ $\left.2.5 \mathrm{ppm}, \mathrm{H}_{2} \mathrm{O}_{2}=10 \mathrm{ppm}\right]$. 
Se determino la relación óptima de la $[\mathrm{Fe} 2+/ \mathrm{H} 2 \mathrm{O} 2]$ a través de experimentos con las relaciones de $\left[\mathrm{Fe}^{2+} / \mathrm{H}_{2} \mathrm{O}_{2}\right]$ de $(0.5 / 2) \mathrm{mg} / \mathrm{L}$ y $(2.5 / 10) \mathrm{mg} / \mathrm{L}$, en presencia de radiación UV-A $\mathrm{y}$ en un tiempo de 60 minutos.

La relación $\left[\mathrm{Fe}^{2+} / \mathrm{H}_{2} \mathrm{O}_{2}\right]=2.5 / 10$ ha degradado casi el doble del porcentaje que la relación $\left[\mathrm{Fe}^{2+} / \mathrm{H}_{2} \mathrm{O}_{2}\right]=0.5 / 2$ (Figura 4), esto a causa de la disponibilidad de concentración inicial del catalizador hierro y de peróxido de hidrógeno. Tal como lo demuestra Tolba et al., 2019'11, la ausencia de peróxido de hidrógeno retrasa la reacción alcanzando solo un $37.5 \%$ de degradación; en cuanto se aumenta las dosis de $1 \mathrm{mg} / \mathrm{L}$ a $1.5 \mathrm{mg} / \mathrm{L}$ a $2 \mathrm{mg} / \mathrm{L}$ la eficiencia incrementa directamente, sin embargo, se confirmó que la dosis $2 \mathrm{mg} / \mathrm{L}$, es un excedente que no mejoro la eficacia del proceso.

Por ello, una relación estequiométrica directa entre la concentración inicial de metomilo y la de $\mathrm{H}_{2} \mathrm{O}_{2}$ tal como se desarrolla en la presente investigación, ha efectuado una degradación eficiente del metomilo, sin tener la necesidad de incrementar la concentración de peróxido de hidrógeno. En tanto la fotoreducción del $\mathrm{Fe}^{3+}$ y la formación de radical $\bullet \mathrm{OH}$ se da con la irradiación de luz, acelerando el proceso como muestra la ecuación 2 y $3^{12}$.

$$
\begin{aligned}
& \mathrm{Fe}^{2+}+\mathrm{H}_{2} \mathrm{O}_{2} \rightarrow \mathrm{Fe}(\mathrm{OH})^{2+}+\cdot \mathrm{OH} \\
& \mathrm{Fe}(\mathrm{OH})^{2+}+\mathrm{h} v \rightarrow \mathrm{Fe}^{2+}+\cdot \mathrm{OH}
\end{aligned}
$$

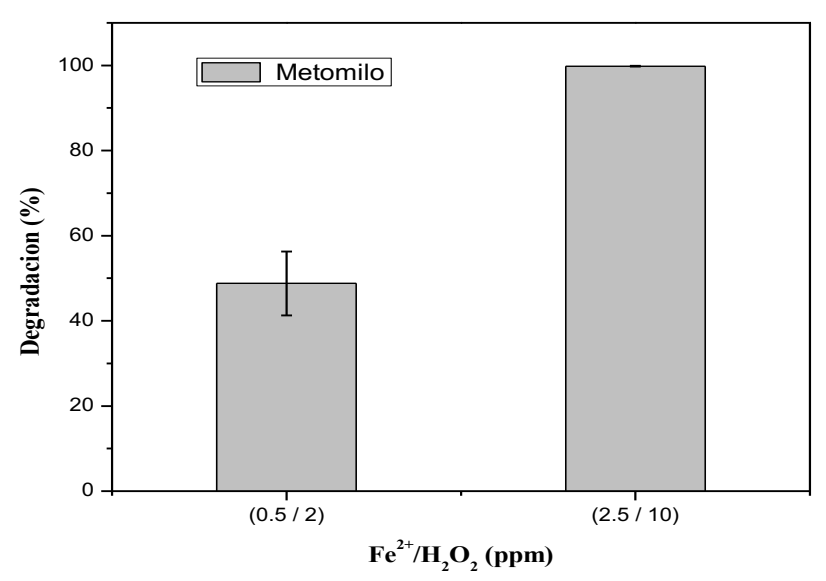

Figura 4. Determinación de la relación de $\mathrm{Fe}^{2+} / \mathrm{H}_{2} \mathrm{O}_{2}$ Óptima para la degradación de metomilo, bajo condiciones experimentales $\left[\mathrm{pH}=3.6, \mathrm{H}_{2} \mathrm{O}_{2}=10 \mathrm{ppm}, \mathrm{t}=60 \mathrm{~min}\right]$.

En la determinación del $\mathrm{pH}$ optimo, se evaluó la degradación de metomilo en medio ácido y neutro, durante un tiempo óptimo de 60 minutos, con la relación de $\left[\mathrm{Fe}^{2+} / \mathrm{H}_{2} \mathrm{O}_{2}\right]=2.5 / 10 \mathrm{mg} / \mathrm{L}$ y en presencia de radiación UV-A. 
Buitrago et al., $2000^{13}$ reporta que el proceso foto-Fenton trabaja mejor a $\mathrm{pH}$ ácido, ya que bajo estas condiciones se generan acuacomplejos férricos altamente fotoactivos bajo radiación UV-A, siendo esta una variable significativa para el proceso, verificado en la presente investigación (Figura 5), en la que se utilizó un pH de 3.6 y obtuvo una degradación del $100 \%$, demostrando que este $\mathrm{pH}$ puede ser el óptimo para la degradación de metomilo bajo estas condiciones experimentales. En tanto se ha demostrado en los resultados que a $\mathrm{pH}$ 7 , solo se pude degradar un $3.68 \%$ de metomilo, esto se debe a que se forman óxidos de hierro que no son fotoactivos ${ }^{14}$.

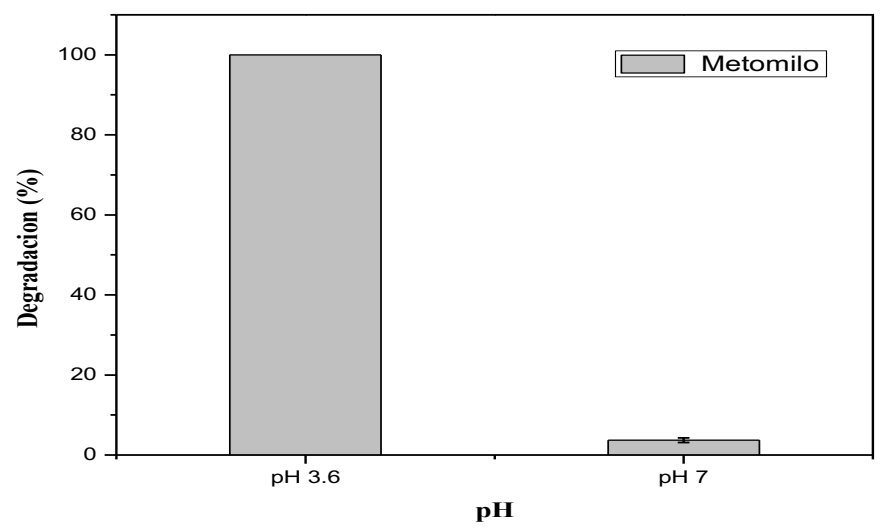

Figura 5. Determinación del pH óptimo para la degradación de metomilo.

\section{Proceso $\mathrm{H}_{2} \mathrm{O}_{2} / \mathrm{UV}$}

El tiempo de irradiación optimo se determinó mediante el registro de la degradación de metomilo bajo irradiación UV-C, usando una solución de $\mathrm{H}_{2} \mathrm{O}_{2} 10 \mathrm{mg} / \mathrm{L}$ (Figura 6). Los resultados obtenidos revelan una rápida degradación del insecticida luego de 4 minutos de radiación.

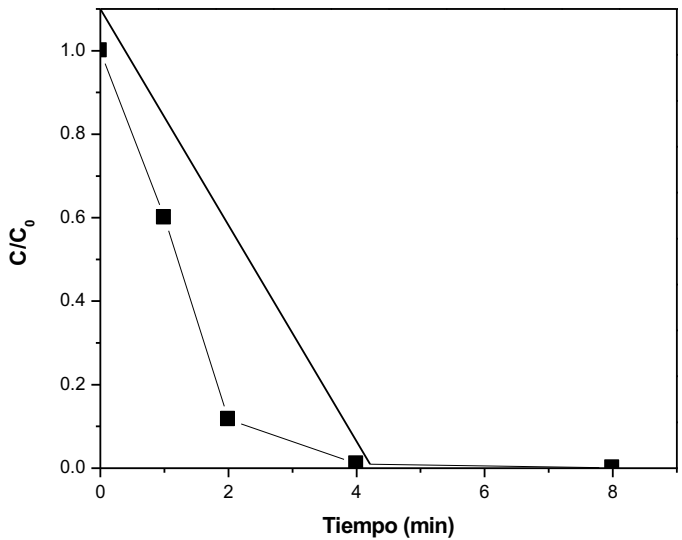

Figura 6. Gráfico $\mathrm{C} / \mathrm{C} 0$ de metomilo vs tiempo en el último experimento de tiempo para la determinación del tiempo de irradiación. 
Los resultados de la determinación de la concentración de peróxido de hidrógeno, donde se consideraron las concentraciones de $3 \mathrm{mg} / \mathrm{L}$ y $10 \mathrm{mg} / \mathrm{L}$ (Tabla 4) revelan que a mayor concentración de $\mathrm{H}_{2} \mathrm{O}_{2}$, mayor degradación del ingrediente activo. Para este montaje experimental, la concentración de $\mathrm{H}_{2} \mathrm{O}_{2}$ que logro mayor degradación fue de $\mathrm{mg} / \mathrm{L}$. Es posible lograr una mayor degradación a mayores concentraciones de $\mathrm{H}_{2} \mathrm{O}_{2}$, sin embargo, es importante considerar el incremento de costos, así como los efectos ambientales probables.

Tabla 4. Resultados del experimento para la determinación de la concentración de peróxido de hidrógeno.

\begin{tabular}{cccc}
\hline $\mathbf{H}_{2} \mathbf{O}_{2}$ & Tiempo (min) & $\begin{array}{c}\text { Concentración de } \\
\text { metomilo residual (mg/L) }\end{array}$ & Degradación \\
\hline $3 \mathrm{ppm}$ & 5 & 0.3 & $90.99 \%$ \\
$10 \mathrm{ppm}$ & 5 & 0.06 & $98.20 \%$ \\
\hline
\end{tabular}

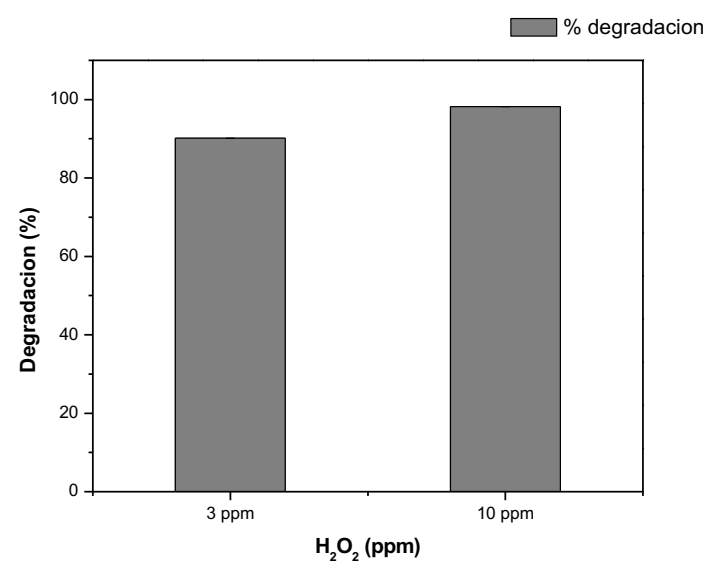

Figura 7. Comparación de la degradación de metomilo con dos diferentes concentraciones de peróxido de hidrógeno.

Sobre la influencia del tipo de radiación ultravioleta dentro del proceso $\mathrm{H}_{2} \mathrm{O}_{2} / \mathrm{UV}$, se realizaron experimentos con tres tipos de radiación ultravioleta UV-A, UV-B y UV-C (Tabla 5). El experimento de control de fotolisis del metomilo bajo luz UV-A y UV-B (en ausencia de peróxido de hidrogeno) reveló una baja degradación debido a que el metomilo presenta un máximo de absorción de luz a $235 \mathrm{~nm}$ (luz UV-C). Mientras que bajo radiación UV-C si se observó una degradación del insecticida del $99.57 \%$ debido a procesos de fotolisis de la molécula. En presencia de peróxido de hidrogeno $(10 \mathrm{mg} / \mathrm{L})$ y radiación UV-A, se observó una leve degradación producida probablemente a una incipiente del $\mathrm{H}_{2} \mathrm{O}_{2}$, mientras que bajo UV-B no se observó mejora alguna. En condiciones de radiación con UV-C, se evidenció una rápida cinética de degradación debido al efecto de procesos altamente efectivos de fotolisis del $\mathrm{H}_{2} \mathrm{O}_{2}$ y de la molécula del pesticida. 
Tabla 5. Resultados del sexto experimento para la determinación del tipo de irradiación.

\begin{tabular}{ccccc}
\hline Radiación & Tiempo & $\mathbf{H}_{\mathbf{2}} \mathbf{O}_{\mathbf{2}} \mathbf{( p p m )}$ & Metomilo $\mathbf{( p p m )}$ & Degradación \\
\hline Sin UV & 0 & 0 & 10 & $0.00 \%$ \\
UV-A & 5 & 10 & 9.23 & $7.67 \%$ \\
UV-B & 5 & 10 & 10 & $0.00 \%$ \\
UV-C & 5 & 10 & 0.043 & $99.57 \%$ \\
\hline
\end{tabular}

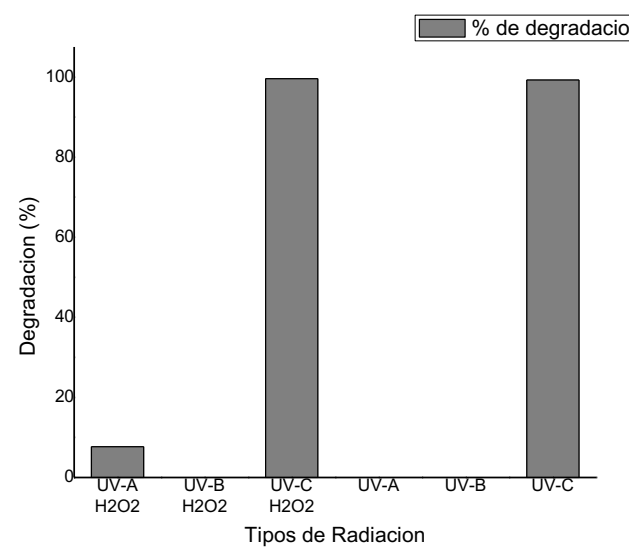

Figura 8. Comparación de la degradación de metomilo con diferentes tipos de radiación ultravioleta vs controles sin peróxido de hidrógeno.

\section{Proceso de Ozonización}

Se determinó la concentración de ozono alcanzando valores de 0.07 y $0.08 \mathrm{mg} \mathrm{O} / \mathrm{L}$ en los

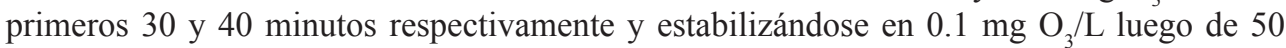
minutos.

Tabla 6. Concentración de ozono en diferentes tiempos.

\begin{tabular}{cc}
\hline Tiempo $(\mathbf{m i n})$ & Concentración $(\mathbf{m g} / \mathbf{L})$ \\
\hline $\mathbf{1 0}$ & 0.07 \\
$\mathbf{2 0}$ & 0.07 \\
$\mathbf{3 0}$ & 0.07 \\
$\mathbf{4 0}$ & 0.08 \\
$\mathbf{5 0}$ & 0.10 \\
\hline
\end{tabular}


Para determinar las condiciones óptimas de pH y tiempo en la ozonización para la degradación del metomilo, a través del equipo de ozonización se generó una concentración $0.07 \mathrm{mg} / \mathrm{L}$ correspondiente a un flujo de $0.5 \mathrm{~L} \mathrm{~min}^{-1}$ de oxígeno. Se adicionó un experimento control, para determinar el efecto de hidrolisis sobre la degradación de metomilo en los mismos tiempos de exposición del pH 11.

La degradación de metomilo en el proceso de ozonización a pH 8, en 30 min es del $0 \%$ (Tabla 7). A pH 11 por el contrario, se observó una rápida y total degradación del contaminante luego de 10 min de tratamiento. El experimento de control a pH 11 (en ausencia de ozono) reveló que no hubo participación alguna de procesos de hidrolisis en la degradación del metomilo, demostrando que la acción generadora de radicales hidroxilos por parte del proceso de ozonización a $\mathrm{pH}$ alcalino fue la responsable de la desaparición del agroquímico. La degradación de metomilo a pH 11 y control del pH 11, donde el experimento control a pH 11 es la degradación de metomilo sin someter al proceso de ozonización, muestra que no se tiene ningún efecto de hidrolisis de metomilo, y que el metomilo no es inestable o fácil de ser descompuesto en esos tiempos.

Se verifico que, en pH alcalino, el proceso de ozonización del metomilo es más eficiente como se aprecia en la Tabla 7. Se muestra que en el tiempo 4 min una concentración de $6.55 \mathrm{mg} / \mathrm{L}$ de metomilo, se alcanza una degradación de $34.5 \%$. Se obtuvo una total degradación del metomilo cuando el tiempo de ozonización se prolongó a 10 min, teniendo una concentración de $0.4 \mathrm{mg} / \mathrm{L}$, siendo degradado el $96 \%$. La concentración de metomilo disminuye significativamente cuando el valor de $\mathrm{pH}$ aumenta a 11, debido a que en este $\mathrm{pH}$ el ozono disuelto es descompuesto para formar radicales hidroxilos y oxida el efluente ${ }^{8}$.

Al finalizar el experimento se concluyó que la ozonización es efectiva a pH 11 y tiempo de 10 min, ya que se observa una mayor degradación considerando estas las condiciones óptimas para aplicar la degradación del metomilo, tanto en agua simulada y real.

Tabla 7. Degradación de metomilo a pH 8, $\mathrm{pH} 11$ y control para $\mathrm{pH}$ 11: flujo $0.5 \mathrm{~L} / \mathrm{min}$, $\left[\mathrm{O}_{3}\right]=0.07 \mathrm{mg} / \mathrm{L}$.

\begin{tabular}{cc|cc|cc}
\hline \multicolumn{2}{c|}{ pH 8 } & \multicolumn{2}{c|}{ pH 11 } & \multicolumn{2}{c}{ Control para pH 11 } \\
\hline $\begin{array}{c}\text { Tiempo } \\
\text { (min) }\end{array}$ & $\begin{array}{c}\text { \% } \\
\text { Degradación }\end{array}$ & $\begin{array}{c}\text { Tiempo } \\
\text { (min) }\end{array}$ & $\begin{array}{c}\text { \% } \\
\text { Degradación }\end{array}$ & $\begin{array}{c}\text { Tiempo } \\
\text { (min) }\end{array}$ & $\begin{array}{c}\% \\
\text { Degradación }\end{array}$ \\
\hline 0 & 0 & 0 & 0 & 0 & 0 \\
5 & 0 & 2 & 0 & 2 & 0 \\
10 & 0 & 4 & 34.5 & 4 & 0 \\
15 & 0 & 8 & 72.2 & 8 & 0 \\
30 & 0 & 10 & 96.0 & 10 & 0 \\
\hline
\end{tabular}

\subsection{Degradación de metomilo en Agua Simulada Proceso foto-Fenton}

Para determinar el efecto de los iones en la degradación del metomilo, se usaron las condiciones óptimas de $3.6 \mathrm{de} \mathrm{pH}$, así como 2.5 y $10 \mathrm{mg} / \mathrm{L}$ de $\mathrm{Fe} / \mathrm{H}_{2} \mathrm{O}_{2}$ y tiempo de 60 minutos, obtenidas en el experimento realizado en agua destilada, por esta razón no se realizaron repeticiones con diferentes condiciones. 
Para definir la acción de los aniones en la degradación de metomilo, se realizaron experimentos con el promedio de las concentraciones encontradas en el análisis de aguas provenientes de los drenes, considerando, Fluoruro $\left[\mathrm{F}^{-}\right]=0.30 \mathrm{mg} / \mathrm{L}$, Nitrato $\left[\mathrm{NO}_{3}^{-}\right]=5 \mathrm{mg} / \mathrm{L}$ y Carbonato $\left[\mathrm{CO}_{3}{ }^{2-}\right.$ ] $=20 \mathrm{mg} / \mathrm{L}$, obteniendo los resultados de la degradación de metomilo(Tabla 8 ) en presencia de iones interferentes, indicando la concentración restante de metomilo y su porcentaje de degradación, durante el tiempo de 60 minutos, expuestos a radiación UV-A. Los resultados evidenciaron una reducción de metomilo cercana al 23.14\% luego de 60 min de irradiación bajo concentraciones de ion ferroso y peróxido de hidrogeno de 2.5 y $10 \mathrm{mg} / \mathrm{L}$. Tal como lo demuestra Andreozzi et al $1999^{15}$ al considerar que las especies inorgánicas disueltas de $\mathrm{HCO}_{3}^{-} \mathrm{y} \mathrm{CO}_{3}{ }^{2-}$, aumentan la alcalinidad del agua:

$$
\begin{aligned}
& \mathrm{HCO}_{3}^{-}+\mathrm{H}^{+} \rightarrow \mathrm{H}_{2} \mathrm{O}+\mathrm{CO}_{2} \\
& \mathrm{CO}_{3}{ }^{2-}+2 \mathrm{H}^{+} \rightarrow \mathrm{H}_{2} \mathrm{O}+\mathrm{CO}_{2}
\end{aligned}
$$

Este fenómeno se debe a la sobresaturación del agua con iones carbonatos igual a $20 \mathrm{mg} / \mathrm{L}$. Así mismo, Wang et al., $2019^{16}$ determinó que el aumento del pH generó la precipitación del catalizador de hierro a hidróxido de hierro, limitando la reacción por la menor actividad, siendo este el caso que se presenta en la experimentación en agua simulada. Debido a que el agua de los drenes que se utilizó para la realización de estos experimentos contiene una concentración de aniones bicarbonato de aproximadamente $37 \mathrm{mg} / \mathrm{L}$, se decidió realizar estos experimentos. Así mismo, algunas investigaciones ${ }^{17,18}$ reportan que, en aguas con contenidos importantes de bicarbonato $(>100 \mathrm{mg} / \mathrm{L})$, este anión reacciona con radicales $\mathrm{OH}$ generando un radical menos oxidante (radical bicarbonato) pero igualmente capaz de participar en reacciones de oxidación. De esta manera, pudo confirmarse que a concentraciones inferiores de $100 \mathrm{mg} / \mathrm{L}$ de bicarbonato, este participa más como un secuestrador de radicales $\mathrm{OH}$. La concentración generada de radical bicarbonato no es suficiente para generar un efecto positivo en la degradación.

Por otro lado, Devi et al., $2010^{19}$ evaluó la interferencia de los aniones, $\mathrm{Na}_{2} \mathrm{CO}_{3}, \mathrm{NaHCO}_{3}$, $\mathrm{Na}_{2} \mathrm{SO}_{4}, \mathrm{NaCl}, \mathrm{KNO}_{3}$ para determinar el comportamiento del peróxido de hidrógeno para generar radicales $\bullet \mathrm{OH}$, estableciendo que los aniones carbonato, bicarbonato y nitrato no tienden a formar complejos con el hierro, he ahí la influencia de estos aniones en la baja cinética de degradación, inclusive a mayores concentraciones, por lo que los iones $\mathrm{CO}_{3}^{2}$, $\mathrm{NO}_{3}^{-}$y $\mathrm{HCO}_{3}^{-}$compiten por radicales hidroxilo junto con moléculas del sustrato orgánico a degradar.

Como se muestra en las siguientes reacciones, los aniones carbonato y bicarbonato actúan como secuestradores de radicales hidroxilo generando radicales carbonato con un potencial de oxidación menor ${ }^{15}$.

$$
\begin{aligned}
& \mathrm{HO} \cdot+\mathrm{HCO}_{3-}-\mathrm{CO}_{3}^{-\cdot} \\
& \mathrm{HO} \cdot+\mathrm{CO}_{3}^{2-} \rightarrow \mathrm{CO}_{3}^{-\cdot}+\mathrm{HO}
\end{aligned}
$$


Tabla 8. Resultados de la degradación del metomilo en presencia de iones interferentes (carbonato $\left(\mathrm{CO}_{3}{ }^{-}\right)$, fluoruro $\left(\mathrm{F}^{-}\right)$y nitrato $\left(\mathrm{NO}_{3}^{-}\right)$).

\begin{tabular}{cc|cc|cc}
\hline \multicolumn{2}{c|}{ pH 8 } & \multicolumn{2}{c|}{ pH 11 } & \multicolumn{2}{c}{ Control para pH 11 } \\
\hline $\begin{array}{c}\text { Tiempo } \\
\text { (min) }\end{array}$ & $\begin{array}{c}\text { \% } \\
\text { Degradación }\end{array}$ & $\begin{array}{c}\text { Tiempo } \\
\text { (min) }\end{array}$ & $\begin{array}{c}\text { \% } \\
\text { Degradación }\end{array}$ & $\begin{array}{c}\text { Tiempo } \\
\text { (min) }\end{array}$ & $\begin{array}{c}\% \\
\text { Degradación }\end{array}$ \\
\hline 0 & 0 & 0 & 0 & 0 & 0 \\
5 & 0 & 2 & 0 & 2 & 0 \\
10 & 0 & 4 & 34.5 & 4 & 0 \\
15 & 0 & 8 & 72.2 & 8 & 0 \\
30 & 0 & 10 & 96.0 & 10 & 0 \\
\hline
\end{tabular}

\section{Proceso H2O2/UV}

Los resultados de la aplicación del proceso $\mathrm{H}_{2} \mathrm{O}_{2} / \mathrm{UV}-\mathrm{C}$ en agua simulada (Tabla 9) muestran que al cabo de un tiempo de irradiación de 5 minutos con una concentración de peróxido de hidrógeno de $10 \mathrm{mg} / \mathrm{L}$, la concentración de metomilo se redujo en un 98,99\%. De este modo, la degradación en agua simulada ocurre a pesar de tener la presencia de los iones carbonato, nitrato y cloruro que podrían haber causado interferencia en el proceso de degradación del metomilo. Como se demostró anteriormente, el metomilo sufre principalmente reacciones de fotolisis bajo irradiación UV-C en ausencia o presencia de $\mathrm{H}_{2} \mathrm{O}_{2}$, de esta manera los iones podrían afectar poco su degradación.

Tabla 9. Resultados de la degradación de metomilo en agua simulada.

\begin{tabular}{llll}
\hline Tiempo (min) & $\mathbf{H}_{2} \mathbf{O}_{2}(\mathbf{p p m})$ & Metomilo (ppm) & Degradación \\
\hline 0 & 10 & 20.67 & $0.00 \%$ \\
5 & 10 & 0.22 & $98.99 \%$ \\
\hline
\end{tabular}

\section{Proceso Ozonización}

El resultado de la degradación de metomilo por el proceso de ozonización (Tabla 10) con las condiciones óptimas: concentración de ozono $0.07 \mathrm{mg} / \mathrm{L}, \mathrm{pH} 11$ y tiempo de contacto de $10 \mathrm{~min}$, no muestra una degradación de metomilo en las condiciones óptimas, ya que esta agua simulada contiene aniones interferentes nitratos $\left(\mathrm{NO}_{3}\right)$, carbonatos $\left(\mathrm{CO}_{3}^{-2}\right)$ y cloruros $\left(\mathrm{Cl}^{-}\right)$, lo cual afecta en el proceso de ozonización. La existencia de cloruros conduce a un significante retraso en la ozonización en $\mathrm{pH}$ ácidos (menores a 4), el cloro reacciona con el ozono en condiciones acidas como se muestra en las ecuaciones 8 y $9^{20}$.

$$
\begin{aligned}
& \mathrm{O}_{3}+\mathrm{Cl}^{-} \rightarrow \mathrm{O}_{2}+\mathrm{ClO}^{-} \\
& 2 \mathrm{H}^{+}+\mathrm{Cl}^{-}+\mathrm{ClO}^{-} \leftrightarrow \mathrm{Cl}_{2}+\mathrm{H}_{2} \mathrm{O}
\end{aligned}
$$

Por lo tanto, a pH ácido, los iones cloruros pueden competir eficazmente con el ozono generando especies menos oxidantes ${ }^{20}$. Por otro lado, a un $\mathrm{pH}$ de 7 a más, es decir a $\mathrm{pH}$ alcalinos, baja la concentración de protones desactivando las reacciones anteriores y reduciendo la competencia del cloro con el ozono ${ }^{20}$. Entonces podemos asegurar que, a $\mathrm{pH}$ alcalino, la reacción del cloro con el ozono no interfiere en el proceso de ozonización. 
También se puede evidenciar la existencia de carbonatos, lo cual afecta el proceso. Los iones carbonatos o bicarbonatos a valores de $\mathrm{pH}$ alcalinos son conocidos por su capacidad de secuestrar radicales $\bullet \mathrm{OH}$ en el proceso ozonización reduciendo su eficiencia ${ }^{15,20}$.

Tabla 10. Tratamiento por Ozonización en Agua Simulada: pH 11, tiempo de 10min, flujo $0.5 \mathrm{~L} /$ $\min ,\left[\mathrm{O}_{3}\right]=0.07 \mathrm{mg} / \mathrm{L}$.

\begin{tabular}{ccc}
\hline Tiempo $(\mathbf{m i n})$ & Concentración $(\mathbf{m g} / \mathbf{L})$ & \% Degradación \\
\hline $\mathbf{0}$ & 10 & 0 \\
$\mathbf{1 0}$ & 10 & 0 \\
\hline
\end{tabular}

\subsection{Degradación de metomilo en Agua Real}

\section{Proceso fotofenton}

La muestra de agua obtenida en el dren del P-05, colector final, en el sector agrícola de Uraca-Corire (ARB1-Agua Real Bruta 1), no se encontró presencia de metomilo $(<0.01$ $\mathrm{mg} / \mathrm{L}$, límite de detección de técnica cromatografía liquida MS/MS). Para poder realizar una determinación adecuada de metomilo en aguas reales, se dopó la muestra con una concentración inicial de $10 \mathrm{mg} / \mathrm{L}$. La degradación de metomilo en la matriz de agua real simulado la presencia del ingrediente activo, mediante el proceso foto-Fenton, fue de 94.12 $\%$ bajo un $\mathrm{pH}$ de 3.6. La concentración final alcanzada es de $0.59 \pm 0,13 \mathrm{mg} / \mathrm{L}$ de Metomilo residual.

\section{Proceso $\mathrm{H}_{2} \mathrm{O}_{2} / \mathrm{UV}$}

Bajo irradiación UV-C y presencia de $10 \mathrm{mg} / \mathrm{L}$ de $\mathrm{H}_{2} \mathrm{O}_{2}$ se observó una degradación del $99.38 \%$ de metomilo luego de 5 minutos de experimento (tabla 11), evidenciado que, a pesar de las condiciones complejas de la matriz, el proceso de degradación $\mathrm{H}_{2} \mathrm{O}_{2} / \mathrm{UV}$ es una tecnología efectiva para la degradación del contaminante. Probablemente la presencia de especies inorgánicas u orgánicas en estas aguas reales no absorben luz a longitudes de onda cercanas al máximo de absorción del pesticida $(235 \mathrm{~nm})$ evitando que se genere un efecto protector que evite la fotolisis de la molécula.

Tabla 11. Resultados de la degradación de metomilo en agua real.

\begin{tabular}{cccc}
\hline TIEMPO & $\mathrm{H}_{2} \mathbf{O}_{2}(\mathbf{p p m})$ & METOMILO (ppm) & DEGRADACIÓN \\
\hline 0 & 10 & 27.33 & $0.00 \%$ \\
5 & 10 & 0.17 & $99.38 \%$ \\
\hline
\end{tabular}

\section{Proceso Ozonización}

La degradación de metomilo en agua real por el proceso de ozonización con las condiciones óptimas: concentración de ozono $0.07 \mathrm{mgL}^{-1}$, $\mathrm{pH}$ modificado 11 y tiempo de contacto de 10 min alcanzo una concentración de metomilo de $6 \mathrm{mgL}^{-1}$, teniendo un $40 \%$ de degradación 
en condiciones óptimas. En este tipo de agua no se alcanzó una mayor degradación por la presencia de carbonatos, que secuestra los radicales $\bullet \mathrm{OH}$ reduciendo la eficiencia del proceso de ozonización. Sin embargo, conociendo el complejo del agua real la degradación del metomilo se efectúa, es decir que el tratamiento mediante el proceso de ozonización en medio alcalino puede funcionar en agua reales.

Tabla 12. Tratamiento por Ozonización en Agua Simulada: $\mathrm{pH}$ 11, tiempo de 10min, flujo $0.5 \mathrm{~L} /$ $\min ,\left[\mathrm{O}_{3}\right]=0.07 \mathrm{mg} / \mathrm{L}$.

\begin{tabular}{ccc}
\hline Tiempo (min) & $\mathbf{m g} / \mathbf{L}$ & \%Degradación \\
\hline $\mathbf{0}$ & 10 & 0 \\
$\mathbf{1 0}$ & 6 & 40 \\
\hline
\end{tabular}

\section{CONCLUSIONES}

La tasa de degradación para agua destilada fue de $100 \%$ para el proceso Fotofenton, $99.57 \%$ para el proceso UV-C/ $\mathrm{H}_{2} \mathrm{O}_{2}$ y $96 \%$ para el proceso de ozonización. Para el agua simulada, se alcanzó una degradación máxima de 23.14 \% para el proceso Fotofenton, 98.99 \% para el proceso UV-C/ $\mathrm{H}_{2} \mathrm{O}_{2}$ y $0 \%$ para el proceso de ozonización. Así mismo, para el agua real, se logró una degradación de $94.12 \%$ para el proceso fotofenton, $99.38 \%$ para el proceso $\mathrm{UV}-\mathrm{C} / \mathrm{H}_{2} \mathrm{O}_{2}$ y $40 \%$ para el proceso de ozonización. Los resultados reportados en este trabajo demuestran que los procesos avanzados de oxidación utilizados no fueron tan efectivos en la remoción de metomilo en aguas reales superficiales, principalmente, aquellos procesos en los que la generación de radicales hidroxilos fueron los más afectados por la presencia de la matriz de agua real. Dicha matriz evidenció la presencia de aniones bicarbonato $\left(\mathrm{HCO}_{3}^{-}\right)$que pueden competir por los radicales $\bullet \mathrm{OH}$ fotogenerados generando un radical menos oxidante como lo es el radical carbonilo $\left(\mathrm{CO}_{3} \cdot\right)$ afectando negativamente el proceso de degradación. Es importante considerar que en el proceso UV-C/ $\mathrm{H}_{2} \mathrm{O}_{2}$ también participó un rol importante los procesos vinculados a la fotolisis del metomilo. Por eso razón, la presencia de bicarbonato en el medio no mostró generar un efecto negativo en los procesos $\mathrm{UV}-\mathrm{C} / \mathrm{H}_{2} \mathrm{O}_{2}$. Por su parte, los procesos $\mathrm{UV}-\mathrm{C} / \mathrm{H}_{2} \mathrm{O}_{2}$ parecieron inmunes a la presencia de matriz inorgánica u orgánica en las aguas reales. Esto se debió a que el metomilo bajo radiación UV-C mostró una fuerte reacción de fotolisis. La presencia de $\mathrm{H}_{2} \mathrm{O}_{2}$ y su concomitante generación de radicales $\bullet \mathrm{OH}$ fotogenerados tuvo un efecto menor. De esta manera, la presencia de la matriz inorgánica que probablemente absorbe luz a longitudes de onda de mayor o menor energía que el metomilo no generó un efecto protector o de pantalla que evitara la degradación del pesticida.

\section{AGRADECIMIENTOS}

Los autores agradecen a la Universidad Nacional de San Agustín de Arequipa por el financiamiento brindado por fondos concursables, al cual la presente investigación ha sido seleccionada y ejecutada mediante $\mathrm{N}^{\circ}$ de Contrato de Financiamiento IBA-0038-2017-UNSA 


\section{REFERENCIAS BIBLIOGRÁFICAS}

1. Iannacone J, Alvariño L. Efecto ecotoxicológico del metomilo en Corydoras Lacépède, 1803 (Siluriformes: callichthyidae) y su caracterización leucocitaria. Ecol Apl. 2008;7(1-2):55-61.

2. Lin Z, Zhang W, Pang S, Huang Y, Mishra S, Bhatt P, Chen S. Current Approaches to and Future Perspectives on Methomyl Degradation in Contaminated Soil/Water Environments. Molecules. 2020;25(3):738. doi: 10.3390/molecules25030738.

3. Tomašević A, Kiss E, Petrović S, Mijin D. Study on the photocatalytic degradation of insecticide methomyl in water. Desalination. 2010;262(1-3):228-234.

4. Tony MA, Purcell PJ, Mansour SA. Photodegradation and Box-Behnken design optimization for methomyl using Fenton process based on synthesized $\mathrm{CuO}$ nanocrystals via facile wet chemical technique. Chem Eng Commun. 2020;208(3):349-363.

5. Tomašević A, Mijin D, Gašic S, Kiss E. The influence of polychromatic light on methomyl degradation in $\mathrm{TiO} 2$ and $\mathrm{ZnO}$ aqueous suspension. New pub Balaban. 2014 [cited 2021 Aug 23];52(22-24):4342-4349.

6. Juang RS, Chen $\mathrm{CH}$. Comparative study on photocatalytic degradation of methomyl and parathion over UV-irradiated $\mathrm{TiO} 2$ particles in aqueous solutions. J Taiwan Inst Chem Eng. 2014;45(3):989-995.

7. Mierzwa JC, Rodrigues R, Teixeira ACSC. UV-Hydrogen Peroxide Processes. Adv Oxid Process Wastewater Treat Emerg Green Chem Technol. 2018;13-48.

8. Chang CC, Trinh C, Chiu CY, Chang CY, Chiang SW, Ji DR, et al. UV-C irradiation enhanced ozonation for the treatment of hazardous insecticide methomyl. J Taiwan Inst Chem Eng. 2015;49:100-104.

9. Bader H, Hoigné J. Determination of ozone in water by the indigo method. Water Res. 1981;15(4):449-56.

10. UNE. UNE-EN 15662:2009 Alimentos de origen vegetal. Determinación de residuos de plaguicidas utilizando GC-MS y/o LC-MS /MS seguido de extracción/división de acetonitrilo y método de purificación dispersiva SPE-QuEChERS [Internet]. Normalizacion Española. UNE; 2009 [citado 1 sep 2021]. Disponible en: https://www. une.org/encuentra-tu-norma/busca-tu-norma/norma?c=N0043515

11. Tolba A, Gar Alalm M, Elsamadony M, Mostafa A, Afify H, Dionysiou DD. Modeling and optimization of heterogeneous Fenton-like and photo-Fenton processes using reusable Fe3O4-MWCNTs. Process Saf Environ Prot. 2019;128:273-283.

12. Sreeja PH, Sosamony KJ. A Comparative Study of Homogeneous and Heterogeneous Photo-fenton Process for Textile Wastewater Treatment. Procedia Technol. 2016;24:217223.

13. Buitrago JL, Sanabria J, Gútierrez-Zapata HM, Urbano-Ceron FJ, García-Barco A, Osorio-Vargas $\mathrm{P}$, et al. Photo-Fenton process at natural conditions of $\mathrm{pH}$, iron, ions, and humic acids for degradation of diuron and amoxicillin. Environ Sci Pollut Res. 2019;27(2):1608-1624.

14. Vorontsov A V. Advancing Fenton and photo-Fenton water treatment through the catalyst design. J Hazard Mater. 2019;372:103-112. 
15. Andreozzi R, Caprio V, Insola A, Marotta R. Advanced oxidation processes (AOP) for water purification and recovery. Catal Today. 1999;53(1):51-59.

16. Wang J, Zhang Q, Deng F, Luo X, Dionysiou DD. Rapid toxicity elimination of organic pollutants by the photocatalysis of environment-friendly and magnetically recoverable step-scheme SnFe2O4/ZnFe2O4 nano-heterojunctions. Chem Eng J. 2020;379:122264. doi: 10.1016/j.cej.2019.122264.

17. Alvear-Daza JJ, García-Barco A, Osorio-Vargas P, Gutiérrez-Zapata HM, Sanabria $\mathrm{J}$, Rengifo-Herrera JA. Resistance and induction of viable but non culturable states (VBNC) during inactivation of E. coli and Klebsiella pneumoniae by addition of $\mathrm{H} 2 \mathrm{O} 2$ to natural well water under simulated solar irradiation. Water Res. 2021;188:116499. doi: 10.1016/j.watres.2020.116499.

18. Gutiérrez-Zapata HM, Rojas KL, Sanabria J, Rengifo-Herrera JA. 2,4-D abatement from groundwater samples by photo-Fenton processes at circumneutral $\mathrm{pH}$ using naturally iron present. Effect of inorganic ions. Environ Sci Pollut Res. 2016;24(7):6213-6221.

19. Devi LG, Raju KSA, Kumar SG, Rajashekhar KE. Photo-degradation of di azo dye Bismarck Brown by advanced photo-Fenton process: Influence of inorganic anions and evaluation of recycling efficiency of iron powder. J Taiwan Inst Chem Eng. 2011;42(2):341-349.

20. Rao YF, Chu W. Degradation of linuron by UV, ozonation, and UV/O3 processesEffect of anions and reaction mechanism. J Hazard Mater. 2010;180(1-3):514-523. 